\title{
BMJ Open What factors are important for deprescribing in Australian long-term care facilities? Perspectives of residents and health professionals
}

\author{
Justin P Turner, ${ }^{1,2}$ Susan Edwards, ${ }^{3}$ Melinda Stanners, ${ }^{4}$ Sepehr Shakib, ${ }^{2,5}$ \\ J Simon Bell ${ }^{1,6}$
}

To cite: Turner JP, Edwards S, Stanners M, et al. What factors are important for deprescribing in Australian long-term care facilities? Perspectives of residents and health professionals. BMJ Open 2016:6:e009781. doi:10.1136/bmjopen-2015009781

- Prepublication history for this paper is available online. To view these files please visit the journal online (http://dx.doi.org/10.1136/ bmjopen-2015-009781)

Received 21 August 2015 Revised 2 January 2016 Accepted 29 January 2016

CrossMark

For numbered affiliations see end of article.

Correspondence to Justin P Turner; justin.turner@monash.edu

\section{ABSTRACT}

Objectives: Polypharmacy and multimorbidity are common in long-term care facilities (LTCFs). Reducing polypharmacy may reduce adverse events and maintain quality of life. Deprescribing refers to reducing medications after consideration of therapeutic goals, benefits and risks, and medical ethics. The objective was to use nominal group technique (NGT) to generate then rank factors that general medical practitioners (GPs), nurses, pharmacists and residents or their representatives perceive are most important when deciding whether or not to deprescribe medications.

Design: Qualitative research using NGT.

Setting: Participants were invited if they worked with, or resided in LTCFs across metropolitan and regional South Australia.

Participants: 11 residents/representatives, 19 GPs, 12 nurses and 14 pharmacists participated across six separate groups.

Methods: Individual groups of GPs, nurses, pharmacists and residents/representatives were convened. Using NGT each group ranked factors perceived to be most important when deciding whether or not to deprescribe. Then, using NGT, the prioritised factors from individual groups were discussed and prioritised by a multidisciplinary metropolitan and regional group comprised of resident representatives, GPs, nurses and pharmacists.

Results: No two groups had the same priorities. GPs ranked 'evidence for deprescribing' and

'communication with family/resident' as most important factors. Nurses ranked 'GP receptivity to deprescribing' and 'nurses ability to advocate for residents' as most important. Pharmacists ranked 'clinical appropriateness of therapy' and 'identifying residents' goals of care' as most important. Residents ranked 'wellbeing of the resident' and 'continuity of nursing staff' as most important. The multidisciplinary groups ranked 'adequacy of medical and medication history' and 'identifying residents' goals of care' as most important. Conclusions: While each group prioritised different factors, common and contrasting factors emerged. Future deprescribing interventions need to consider the similarities and differences within the range of factors prioritised by residents and health professionals.
Strengths and limitations of this study

- This is which first study to consider which factors are important for deprescribing from the perspectives of general practitioners, nurses, pharmacists, and residents and their representatives.

- Participants were drawn for a large geographical area to provide maximum variation of opinions and experiences.

- A structured evaluative methodology was used allowing groups to generate and prioritise factors important for deprescribing.

- A limitation was that the results may not be generalisable to other countries where aged care services are structured differently. Recruitment by invitation may make the sample biased towards motivated and enthusiastic participants with an interest in deprescribing. This was a strength because it enables a rich discussion of factors important for deprescribing. However, it is unclear whether less motivated participants perceive similar factors as important.

\section{INTRODUCTION}

Older people living in long-term care facilities (LTCFs) often have multimorbidity. ${ }^{1}$ Between $38 \%$ and $91 \%$ of residents take $\geq 5$ medications. $^{2}$ As people age the benefit-to-risk ratio of medications may change, and this may mean that medications that were once prescribed appropriately become inappropriate. ${ }^{3}$ Polypharmacy in older people is associated with hospitalisations, adverse drug events (ADEs), drug-drug interactions, drugdisease interactions, falls, impaired functional capacity and cognitive impairment. ${ }^{4-7}$

Deprescribing refers to the stepwise reduction of unnecessary or potentially inappropriate medications (PIMs) after consideration of therapeutic goals, benefits and risks, and medical ethics. ${ }^{8-11}$ One goal of deprescribing is to reduce the negative outcomes associated 
with polypharmacy. ${ }^{12}$ Preliminary research in older people suggests that many medications can be withdrawn safely, resulting in global improvements in patient perceived health, reduced hospitalisations and increased longevity. ${ }^{13}{ }^{14}$ Despite this, over $50 \%$ of LTCF residents take PIMs. ${ }^{15} 16$

Previous research has focused on family physician/ general medical practitioner (GP) perspectives on deprescribing for community-dwelling patients, ${ }^{17}$ and residents of LTCFs. ${ }^{18}{ }^{19}$ Prescribers barriers to deprescribing include lack of awareness of the need to deprescribe, prescribing inertia, self-efficacy and limited feasibility due to external factors. ${ }^{20}$ Patients' barriers to deprescribing include lack of an established process, uncertainty regarding the appropriateness, and fear of medication cessation. Families have been identified as possible barriers or enablers to deprescribing, while patient 'dislike' of medications has been considered an enabler. $^{21}$

Prescribing decisions made by GPs may be influenced by nurses, pharmacists, other medical specialists and residents or their representatives. ${ }^{22}$ Each stakeholder may perceive different factors to be important when deciding whether deprescribing is warranted. Furthermore, organisational culture within LTCFs can influence medication use. ${ }^{23}$ Successful deprescribing is likely to be dependent on agreed priorities that consider the perspectives of multiple disciplines and the resident. $^{24}{ }^{25}$ Studies to date have focused on single groups such as GPs or community-dwelling patients, ${ }^{25} 26$ whereas no studies have investigated the factors important to multiple disciplines and residents when considering deprescribing in LTCFs, either as individual groups, or in a multidisciplinary setting.

Nominal group technique (NGT) and the Delphi technique are the two most frequently used methods for arriving at a group consensus. ${ }^{27-29}$ NGT is a structured evaluative methodology used to generate and prioritise ideas. $^{28}$ 30-32 NGT encourages a wider generation of ideas than conventional discussion groups, ${ }^{31}$ and facilitates inclusion of all participants. ${ }^{30}$

\section{AIM}

The aim of the study was to rank factors that GPs, nurses, pharmacists and residents perceive as most important when deciding whether or not to deprescribe medications.

\section{METHODS}

\section{Methodological approach}

NGT has been employed to analyse healthcare problems, ${ }^{33}$ in guideline development ${ }^{34}$ and to identify priorities in primary care. ${ }^{32}{ }^{35-37}$ The NGT was chosen over Delphi technique as NGT involves participants in generating their own ideas, while also allowing participants to hear and understand the perspectives of the collective group. The use of a facilitator to moderate group dynamics, as well as the use of anonymous ranking, ensures no one individual is able to dominate the group's outcome. ${ }^{30}$

This study employed two phases (figure 1). In phase 1 , the factors most important for deprescribing according to individual groups of health professionals and residents and their representatives were generated. Phase 2 utilised. Mixed groups comprising of healthcare professionals and residents representatives.

The factors most important for deprescribing according to individual groups of GPs, nurses, pharmacists and residents and or their representatives were generated in phase 1. We hypothesised that groups including residents' representatives, GPs, nurses and pharmacists (multidisciplinary groups) may identify different priorities. Therefore, phase 2 involved two multidisciplinary NGT groups comprising 2-3 residents' representatives, GPs, nurses and pharmacists. These groups discussed the prioritised factors from phase 1 to generate a multidisciplinary ranked list of important factors considered when deprescribing in LTCFs. One multidisciplinary group was convened in a metropolitan area and the other in a regional area. An independent experienced facilitator was present for all sessions. Each NGT session produced a prioritised list of important factors when considering deprescribing in LTCFs.

\section{Study setting and participants}

NGT sessions were conducted with groups of health professionals, permanent residents of LTCFs, as well as residents' representatives. Residents' representatives were invited to ensure the views of residents with cognitive impairment were included. To ensure the maximum variation of opinions and experiences, participants were recruited and specifically selected from across a large geographical area of South Australia including the city of Adelaide, and the Fleurieu peninsular regional area. Furthermore, participants were recruited to provide a broad range of ages, and length of contact with LTCFs. To reduce potential reluctance to voice opinions in front of work colleagues or caregivers, groups were formulated to include participants from a variety of workplaces including different GP family practices, LTCFs, community pharmacies and independent consultant pharmacists. ${ }^{29}$ Groups were designed with approximately 10 participants to maintain group dynamics. ${ }^{28}$ GPs who routinely care for residents in LTCFs were invited to participate. Specialist geriatricians were not invited to participate because GPs, rather than geriatricians, are responsible for the majority of prescribing within Australian LTCFs. Pharmacists who supply medications to and/or have accreditation to provide LTCF medication reviews were also invited to participate. GP and pharmacist potential participants were identified by external independent health professional support organisations. These organisations included GP and pharmacist professional organisations along with government-funded regional organisations 


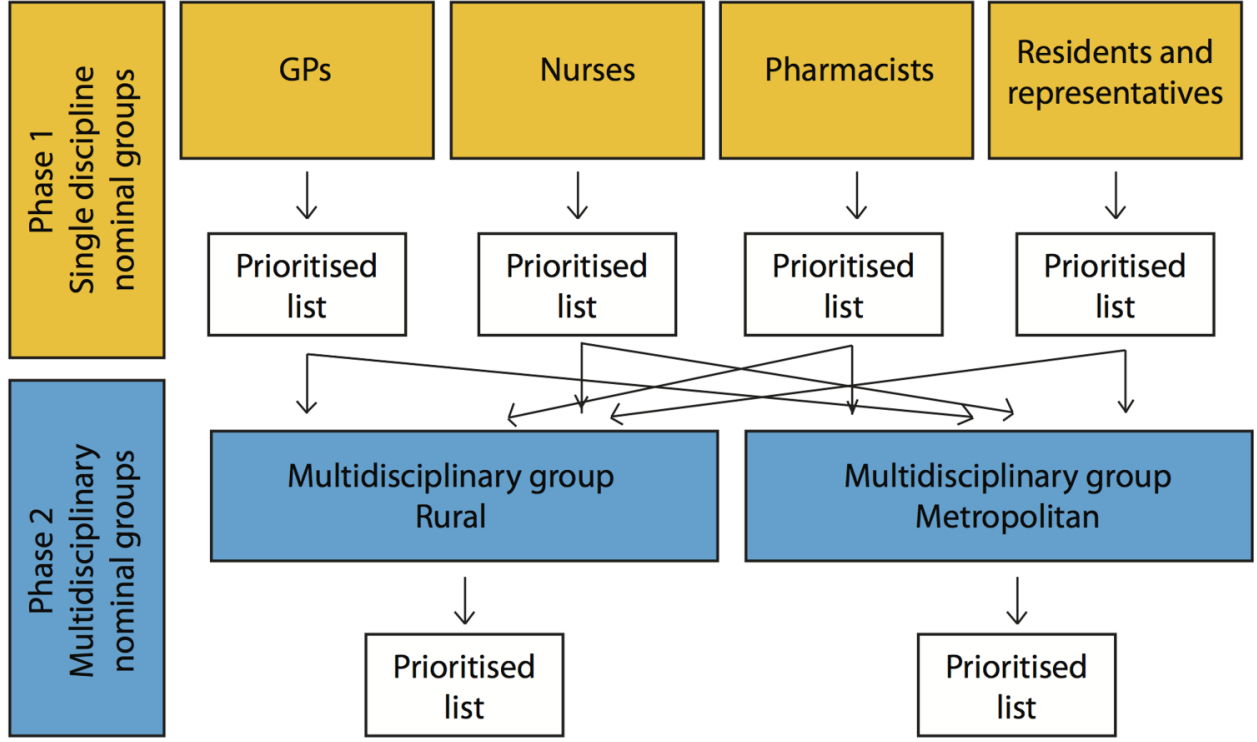

Figure 1 Flow diagram for phase 1 and phase 2. GP, general medical practitioner.

responsible for the provision of professional development and education to health professionals. To provide a range of experience and training levels potential nurse participants were purposefully selected from various LTCFs by an organisation that provides aged care services. The organisations also conducted purposeful recruitment of residents such that the diversity of the group was maximised according to comorbidities, functional status, cognitive status and length of time in LTCFs. Purposeful recruitment also allowed residents representatives to be present on behalf of residents with cognitive decline including dementia or Alzheimer's disease. Residents who had difficulty reading or writing were provided with assistance during the NGT session. To maximise variation of opinions and experiences, participants from phase 1 were not included in phase 2. Phase 1 sessions involving GPs and pharmacists, along with both phase 2 NGT sessions were held after business hours in an independent location to allow healthcare professionals to attend after work.

\section{Data collection}

Demographic data were collected from all participants including age group. Residents recorded the number of years they had lived in a LTCF, while health professionals recorded the number of years since initial registration.

\section{Measurements and definitions}

Prior to each NGT, all participants were provided with the same background reading to introduce the concept of deprescribing. Each NGT session was conducted in the same format. This involved an introduction, silent individual generation of factors, discussion, generation of themes and ranking of factors (figure 2). ${ }^{29} 303233 \mathrm{~A}$ facilitator provided the introduction, reflecting what is known from the literature from non-LTCF settings, before asking each NGT group to broadly consider 'What are the important factors that influence your decision whether or not to reduce medications for residents of long term care facilities?' (step 1). For phase 1, participants were given $10 \mathrm{~min}$ silent time to write down all factors they thought were important (step 2). During step 3, the facilitator recorded all ideas on the board until saturation of ideas was reached, before facilitating discussion. Subsequent to the discussion, common themes were generated. Participants then individually and privately selected five factors from the newly generated themes they considered most important and ranked them in priority order from 5 to 1 (step 5). Each participant's points were summed to determine the factors overall rankings, which was then presented to the group (step 6). Brief discussion was led by the facilitator to confirm the prioritised list was an accurate reflection of the group's collective priorities.

In phase 2 after the introduction, participants were provided with the 'top 5' factors from the residents group and each of the discipline-specific groups generated in phase 1. Participants silently considered these for $10 \mathrm{~min}$ (step 2), before being led in a discussion by the facilitator (step 3). Participants were asked to individually and privately rank factors they perceived to be most important as in phase 1 (step 5). The results were reviewed by the group (step 6). To ensure participants viewed the 'top 5' factors without bias, they were presented in alphabetical order without stating which specific group they originated from.

\section{Ethical considerations}

This study was approved by the Monash University Human Research Ethics Committee. Participants provided written informed consent to participate and were given an honorarium for their involvement. 
Figure 2 Process of nominal group technique (NGT).

\section{Step 1: Introduction:}

The facilitator introduced the topic to the group and explained the format of NGT.

\section{Step 2: Silent generation of factors:}

Each participant was asked to silently consider and document their answers to the NGT question.

\section{Step 3: Discussion / round robin recording of factors:}

The facilitator asked each member for one of their factors, which was written on a whiteboard or "sticky wall". Members presented factors until all factors were recorded. At this point, the facilitator promoted discussion on each of the factors listed, and added further factors that were generated.

\section{Step 4: Break and informal discussion:}

This allowed participants to informally discuss the topic between themselves. During this time, the facilitator and researchers conducted preliminary grouping of factors into themes.

Step 5: Generation of themes and ranking:

The facilitator guided the group in determining common themes from the factors that had been generated. Once agreement was reached on the themes, each participant was asked to independently and privately rank the five most important factors, with the most important receiving five points.

\section{Step 6: Final discussion:}

The scores were tallied and presented to the group.

\section{RESULTS}

There were 56 participants across phase 1 and phase 2, with demographic data presented in table 1 .

\section{Phase 1}

High agreement existed within the individual groups, with the 'top 5' factors accounting for $59-68 \%$ of the groups' prioritised vote (GPs 59\%, nurses $65 \%$, pharmacists $68 \%$ and residents $67 \%$ ) (table 2). GPs ranked lack of 'evidence for deprescribing' as the most important factor and 'ability to communicate the need to deprescribe to residents and their family' as second most important. Nurses ranked 'GP receptivity to deprescribing' and their ability to 'advocate for the resident' as the

Table 1 Demographics of nominal group participants

\begin{tabular}{|c|c|c|c|c|}
\hline Group & $\mathbf{n}$ & Male:female & Age median (range) & $\begin{array}{l}\text { Years of experience } \\
\text { Median (range) }\end{array}$ \\
\hline \multicolumn{5}{|l|}{ Phase 1} \\
\hline Residents/representatives & 6 & $3: 3$ & $80-89$ (70-79 to $90+)$ & $3.0(1-14)$ \\
\hline GPs & 13 & $9: 4$ & $50-59(20-29$ to $60-69)$ & $27.0(5-44)$ \\
\hline Nurses & 6 & $2: 4$ & $30-39(20-29$ to $60-69)$ & $9.0(1-30)$ \\
\hline Pharmacists & 9 & $5: 4$ & $40-49(30-39$ to $60-69)$ & $23.0(8-42)$ \\
\hline \multicolumn{5}{|l|}{ Phase 2} \\
\hline Residents/representatives & 5 & $1: 4$ & $60-69(40-49$ to $60-69)$ & No answers given \\
\hline GPs & 6 & $2: 4$ & $40-49(30-39$ to $60-69)$ & $15.5(8-28)$ \\
\hline Nurses & 6 & $0: 6$ & $50-59(20-29$ to $60-69)$ & $23.5(3-39)$ \\
\hline Pharmacists & 5 & $2: 3$ & $50-59(30-39$ to $60-69)$ & $28(13-33)$ \\
\hline \multicolumn{5}{|l|}{ Overall } \\
\hline Residents/representatives & 11 & $4: 7$ & $70-79$ (40-49 to $90+)$ & $3.0(1-14)$ \\
\hline GPs & 19 & $11: 8$ & $50-59(20-29$ to $60-69)$ & $26.5(5-39)$ \\
\hline Nurses & 12 & $2: 10$ & $50-59(20-29$ to $60-69)$ & $13.5(1-39)$ \\
\hline Pharmacists & 14 & $7: 7$ & $50-59(30-39$ to $60-69)$ & $28.0(8-42)$ \\
\hline
\end{tabular}


Table 2 Top seven factors generated per group

\begin{tabular}{l}
\hline Group \\
\hline Residents \\
Well-being of the resident \\
Continuity of nursing staff \\
Feeling of wellness due to medication \\
Burden of medication administration \\
Residents have the right to question their GP \\
Voice of the resident is not heard \\
Respect the GP and do as I am told \\
GPs \\
Evidence for deprescribing \\
Communication with resident/family \\
GP funding \\
Health system structure \\
Adequacy of medical and medication history \\
Fear of deterioration \\
Residents willingness to deprescribe
\end{tabular}

\section{Nurses}

GP receptivity

Nurses ability to advocate for residents

Regular review of medical conditions 3

Understanding of medications by nurse, $\quad 4$

family and resident

Nurses understanding of the residents

medical conditions

Family-support, beliefs and conflicts $\quad 6$

Communication between nurses $\quad 6$

Health literacy of resident and family 6

Pharmacists

Clinical appropriateness of prescribed therapy

Resident's goals of care

Opportunity and funding for pharmacist 3

follow-up monitoring

Resident's frailty status and other medical 4

conditions

Ranking

1

2

3

4

6

6

1

2

3

3

5

5

7

1

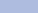

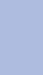

5

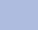

(1)

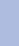

1

1

2

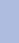

4

Presence of ADEs

Attitudes of prescribers

Burden of medication administration $\quad 7$

Multidisciplinary-regional

Resident's goals of care

Well-being of the resident

Health system structure

Clinical appropriateness of prescribed 4

therapy

Evidence for deprescribing 5

Resident's frailty status and other medical 5

conditions

Regular review of medical conditions

6

7

Multidisciplinary-metropolitan

Adequacy of medical and medication history

Health system structure 2

Evidence for deprescribing 3

Communication with resident/family 4

GP receptivity $\quad 5$

Continuity of nursing staff 6

Resident's goals of care 6

Understanding of medications by nurse, $\quad 6$

family and resident

Some factors received equal scores and are therefore ranked the

same importance.
ADEs, adverse drug events; GP, general medical practitioner. first and second most important factors. Pharmacists ranked 'clinical appropriateness of the prescribed therapy' and 'difficulty in determining the residents' goals of care' as the first and second most important factors. Clinical appropriateness was perceived to include the dose, duration of therapy, class of medication, presence of a suspected or actual ADEs, presence of renal or hepatic impairment and the residents' age and life expectancy. Residents ranked 'wellbeing of the resident' as the most important factor. Residents used this broad heading to include their right to question GPs about medications, to continue medications they believed made them feel well, and cease the medications they perceived contributed to 'burden of medication administration' or caused ADEs. Residents ranked 'continuity of nursing staff' as the second most important factor.

\section{Phase 2}

The 'top 5' factors identified by participants in phase 2 accounted for $52 \%$ of the metropolitan, and $65 \%$ of the rural groups' prioritised vote (table 2). The regional group prioritised a narrow selection of factors, with 7 of the 21 factors presented to them not receiving any votes. The metropolitan group prioritised a wider selection of factors, with five factors not receiving any votes. The regional group ranked the 'residents' goals of care' the highest, while the metropolitan group ranked 'adequacy of medical and medication history' the highest.

\section{DISCUSSION}

This was the first study to rank factors important for deprescribing in LTCFs from the perspectives of health professionals and residents. Previous research has only investigated factors from the perspective of GPs and patients, with a recent review highlighting barriers to deprescribing in various settings. ${ }^{11} 181926$ However, there is a dearth of research investigating barriers to deprescribing from the perspectives of nurses, pharmacists or residents. Although common factors were identified, each NGT group had different priorities. This suggests that successful deprescribing interventions need to recognise and address a range of factors important to residents and health professionals.

Communication was an important similar theme identified by each group. Residents perceived good communication with health professionals is required to achieve 'wellbeing of the resident'. The second highest ranked factor by residents, 'continuity of nursing staff', also related to communication with known and trusted health professionals. Residents commented that unfamiliar nurses were unlikely to be aware of their medical, social and medication history and preferences. This was perceived to potentially result in residents' voices not being heard, which was a barrier to deprescribing. Nurses ranked 'GP receptivity' as their top factor. They identified communicating with GPs, other nurses and the residents' 
family can be difficult. Nurses may have an important role in identifying medications appropriate for deprescribing. ${ }^{38}$ However, in Australia GPs are primarily responsible for care of residents in LTCFs and therefore responsible for altering medication charts. Nurses commented that the success of their recommendations to deprescribe relied on communication with, and receptivity of the GP. While recent research in LTCFs highlights the important role of nurses and organisational culture in prescribing decisions, ${ }^{38}$ our results reveal nurses feel that potentially this important role may be dismissed or not taken seriously.

GPs ranked difficulty communicating the need to deprescribe to residents and families as the second most important factor. They identified a need for guidelines to standardise the process of deprescribing, along with a structured dialogue, to facilitate communicating the benefits of deprescribing with residents and their families. This was consistent with recent Canadian and Dutch research that reported that elucidating patients' preferences and treatment goals was a barrier to deprescribing. ${ }^{25} 39$ Furthermore, GPs reported complexities communicating with specialist physicians. GPs discussed being intimidated by specialist physicians for deprescribing medications they initiated, with one recounting being 'scorned by a colleague'. Furthermore, GPs expressed disappointment when deprescribed medications were restarted by a specialist physician or in hospital. These factors have also been identified in previous research. ${ }^{19} \quad 25 \quad 40 \quad 41$ Likewise, pharmacists recognised these factors as important, with communication underpinning their top two factors.

Similar to the individual groups, factors relating to communication were prioritised by both multidisciplinary groups. However, differences existed between metropolitan and regional views. The metropolitan group prioritised 'adequacy of medical and medication history'. This may reflect residents consulting new health professionals following admission to a LTCF, highlighting inadequate communication between various health professions and residents or their representatives. In contrast, the regional group prioritised the 'residents' goals of care' as most important. It was noted that when residents and GPs had long-established relationships it was challenging to discuss the shift from preventative care to palliative care. This is consistent with previous research. ${ }^{25}$

Reluctance to deprescribe due to 'fear of deterioration' was highly ranked by GPs, and discussed by the pharmacist, resident and multidisciplinary groups. This was consistent with the attitude to deprescribing described by Harriman et $a l^{19}$ 'if it ain't broke, don't fix it'. This concern may be mitigated by gradual individual medication withdrawal, allowing restart if the condition/ symptoms return. ${ }^{21}$ This requires careful ongoing monitoring and good communication. However, all groups raised lack of 'continuity of nursing staff' as a potential barrier to deprescribing. GPs perceived poor continuity of nurse staff limited the opportunity for appropriate monitoring after deprescribing, which may lead to restarting deprescribed medications. Pharmacists perceived lack of 'continuity of nursing staff' limited their ability to determine residents' goals of care. Continuity of nursing staff, or 'consistent assignment,' may improve residents' quality of life, although the evidence is inconsistent. ${ }^{42-45}$

The 'evidence for deprescribing' was the highest ranked factor by the GP group and was prioritised by both multidisciplinary groups. Some participants were either unaware of the evidence or unsure how to apply the results of deprescribing trials to their clinical practice. $^{13} 143846-50$ The lack of evidence was discussed not only in terms of deprescribing but also initiation and continuation of medications in frail older people. Older people are often excluded from clinical trials and clinical practice guidelines may not make recommendations specific to people who are frail or have multimorbidity. A systematic review found $81.3 \%$ of randomised controlled trials excluded patients on the basis of comorbidities, and $38.5 \%$ excluded patients aged $>65$ years. $^{51}$

The factor 'burden of medication administration' was prioritised by the resident group and was similarly discussed by the nurse and pharmacist groups. This included difficulties swallowing large tablets, taste of crushed medications, and use of devices such as inhalers, injections and eye drops. Residents, nurses and pharmacists considered that having a high medication burden was a facilitator of deprescribing.

All groups discussed health system structure concerns including limited funding and incomplete medical and medication histories. Medication histories rarely detail why and when medications were initiated and which prescriber is responsible for ongoing monitoring. All groups agreed that monitoring residents following deprescribing was essential. However, the current health system structure was perceived as not supporting ongoing monitoring.

The 'top-5' highest ranked outcomes from both multidisciplinary groups included 'top 5' factors from most of the individual groups, with each group being represented within the 'top 7.' The multidisciplinary group discussions allowed participants' to consider other points of view, with the final ranking reflecting this discussion. This was most evident with the factor 'evidence for deprescribing' which was ranked highest by the GP group, but was not ranked by the other individual groups. After discussion by residents' representatives, GPs, nurses and pharmacists, it ranked in the 'top 5' for both multidisciplinary groups. Previous research has demonstrated that high-quality multidisciplinary teamwork improves prescribing in LTCFs. ${ }^{24}{ }^{52-54}$ Our results suggest for deprescribing to be effective it is essential to take into consideration the views of residents and individual health professionals. 


\section{Implications for practice}

The results of this study should be considered when instigating interventions to deprescribe in LTCFs. This study highlighted participants uncertainty regarding the evidence for deprescribing at an individual patient level. Interventions that facilitate GPs access to, and dissemination of, the available evidence may facilitate deprescribing. For example, provision of evidence-based guidelines with a structured dialogue may assist communicating with residents and their families regarding deprescribing. Documentation of residents' goals of care may improve decision-making, and ensure the well-being of the resident remains forefront in prescribing decisions. Nurses may benefit from interventions that improve the communication with GPs, and also from educational activities that address their understanding of residents' medications and medical conditions. Interventions that address pharmacists' access to residents' medical information and improve communication between themselves and the family and other disciplines may improve their ability to determine the resident's goals of care. Interventions that improve communication between residents and health professionals, including interventions to improve continuity of nursing staff, may facilitate deprescribing. Furthermore, interventions should consider the priorities of residents and health professionals, both individually and as a collective group.

Although this study was conducted in LTCFs, many factors identified within this study may also be applicable to deprescribing in older people in the broader community setting. It was noted in the regional multidisciplinary NGT that GPs who had long-established relationships with residents found it challenging to discuss the transition from preventative to palliative care. This is likely to be the same for GPs and community-dwelling patients with long-established relationships. As with the LTCF setting, maintaining the patient's well-being at the centre of prescribing decisions is likely to be an important factor for community-dwelling older people.

\section{Strengths and limitations}

Our study included an independent experienced facilitator and a well-defined methodology to elicit a wide range of factors important for deprescribing. Using NGT minimised any pressure that participants may have felt to conform. ${ }^{28}$ Furthermore, participants discussed the final rankings to ensure they were a true reflection of the group. Bias was minimised through using identical background reading material for each group. While this background was based on the current literature relating to deprescribing, it was specifically written in lay terms to account for residents unfamiliar with the subject matter. Participants were recruited from a large geographical area allowing for a range of opinions to be captured. However, the results may not be generalisable to other clinical settings and other countries. Collection of the number of years since registration was a strength of the study as it highlighted the diversity of experience within the each group, however, details relating to specific geriatric training were not collected. It is therefore uncertain if geriatric training impacts on factors considered important for deprescribing. Owing to recruitment by invitation, the sample may be biased towards motivated and enthusiastic participants. This was a strength because it enabled a rich discussion of factors important for deprescribing. However, it is unclear whether less motivated participants perceive similar factors as important. Deprescribing is a complex process which involves many steps. ${ }^{10} 55$ Despite qualitative research having limitations, ${ }^{28}$ qualitatively describing the factors important for the resident and health professionals may facilitate the deprescribing. ${ }^{41}$ However, difference may exist between the expressing of an opinion in a research study and expressing an opinion in a clinical setting. Further research is required to see which factor or group of factors need to be addressed to improve deprescribing in clinical practice.

\section{CONCLUSION}

Using NGT, factors important for deprescribing in LTCFs were identified and prioritised by each disciplinespecific group. Important factors identified include 'evidence for deprescribing', 'GP receptivity to deprescribing', 'clinical appropriateness of therapy' and 'wellbeing of the resident'. The multidisciplinary groups' prioritised both 'adequacy of medical and medication history' and 'identifying residents' goals of care'. Common and contrasting factors were generated between groups, and where similarities existed between groups, they were prioritised differently. Future deprescribing interventions need to recognise and address the similarities and differences within the range of factors prioritised by residents and health professionals.

\section{Author affiliations}

${ }^{1}$ Faculty of Pharmacy and Pharmaceutical Sciences, Centre for Medicine Use and Safety, Monash University, Parkville, Victoria, Australia

${ }^{2}$ Department of Clinical Pharmacology, Royal Adelaide Hospital, Adelaide,

South Australia, Australia

${ }^{3}$ Drug and Therapeutics Service (DATIS), Repatriation General Hospital, Daw Park, South Australia, Australia

${ }^{4}$ Torrens University Australia, Adelaide, South Australia, Australia

${ }^{5}$ Department of Clinical Pharmacology, Faculty of Health Science, University of Adelaide, Adelaide, South Australia, Australia

${ }^{6}$ NHMRC Cognitive Decline Partnership Centre, Hornsby Ku-ring-gai Hospital, University of Sydney, Sydney, New South Wales, Australia

Acknowledgements The authors would like to thank all the participants for their participation in the NGT groups. The author would also like to thank the organisations involved with recruiting appropriate participants, and those who provided the outstanding facilities and catering for the NGT groups. Finally, the authors would like to thank the independent facilitator for her contributions to this research. Preliminary results of this study were presented at the Canadian Geriatrics Society Annual Scientific Meeting, Montreal, Canada, April 2015, the ASCEPT-BPS Joint Scientific Meeting, Hong Kong, China, May 2015, the Pharmaceutical Society of Australia Annual Conference, Sydney, Australia, August 2015, and the ASCEPT-APSA Joint Scientific Meeting, Hobart, Australia, December 2015. 
Contributors JPT contributed to the conception and design of the study. Additionally, JPT was involved with the analysis and interpretation of the results. JPT drafted the manuscript, critically reviewed it and gave final approval of the work. SE contributed to the conception of the study and was involved with the analysis and interpretation of the results. SE critically reviewed the manuscript for important intellectual content and gave her final approval. MS contributed to the design of the study and was involved with the analysis and interpretation of the results. MS critically reviewed the manuscript for important intellectual content and gave her final approval. SS contributed to the conception and design of the study, and assisted with analysis and interpretation of the results. SS critically reviewed the manuscript for important intellectual content and gave his final approval. JSB contributed to the conception of the study, and assisted with interpretation of the results. JSB critically reviewed the manuscript for important intellectual content and gave his final approval. All authors agree to act as guarantor of the work.

Funding JPT was funded through a Faculty Scholarship from the Centre for Medicine Use and Safety, Faculty of Pharmacy and Pharmaceutical Sciences, Monash University.

Competing interests None declared.

Ethics approval Monash University Human Research Ethics Committee.

Provenance and peer review Not commissioned; externally peer reviewed.

Data sharing statement No additional data are available.

Open Access This is an Open Access article distributed in accordance with the Creative Commons Attribution Non Commercial (CC BY-NC 4.0) license, which permits others to distribute, remix, adapt, build upon this work noncommercially, and license their derivative works on different terms, provided the original work is properly cited and the use is non-commercial. See: http:// creativecommons.org/licenses/by-nc/4.0/

\section{REFERENCES}

1. Tinetti ME, Fried TR, Boyd CM. Designing health care for the most common chronic condition-multimorbidity. JAMA 2012;307: 2493-4.

2. Jokanovic N, Tan EC, Dooley MJ, et al. Prevalence and factors associated with polypharmacy in long term care facilities: a systematic review. J Am Med Dir Assoc 2015;16:535.e1-12.

3. Hilmer SN, Gnjidic D, Le Couteur DG. Thinking through the medication list-appropriate prescribing and deprescribing in robust and frail older patients. Aust Fam Physician 2012;41:924-8.

4. Hajjar ER, Cafiero AC, Hanlon JT. Polypharmacy in elderly patients. Am J Geriatr Pharmacother 2007;5:345-51.

5. Maher RL, Hanlon J, Hajjar ER. Clinical consequences of polypharmacy in elderly. Expert Opin Drug Saf 2014;13:57-65.

6. Shah BM, Hajiar ER. Polypharmacy, adverse drug reactions, and geriatric syndromes. Clin Geriatr Med 2012;28:173-86.

7. Tamura BK, Bell CL, Inaba M, et al. Outcomes of polypharmacy in nursing home residents. Clin Geriatr Med 2012;28:217-36.

8. Woodward MC, Bird M, Elliot R, et al. Deprescribing: achieving better health outcomes for older people through reducing medications. J Pharm Pract Res 2003;33:323-7.

9. Reeve E, Turner JP. Patients' perspectives on the brave new word 'deprescribing'. Int J Pharm Pract 2015;23:90-1.

10. Hardy J, Hilmer SN. Deprescribing in the last year of life. J Pharm Pract Res 2011;41:146-51.

11. Garfinkel D, Ilhan B, Bahat G. Routine deprescribing of chronic medications to combat polypharmacy. Ther Adv Drug Saf 2015;6:212-33

12. Thompson $\mathrm{W}$, Farrell $\mathrm{B}$. Deprescribing: what is it and what does the evidence tell us? Can J Hosp Pharm 2013;66:201-2.

13. Garfinkel D, Zur-Gil S, Ben-Israel J. The war against polypharmacy: a new cost-effective geriatric-palliative approach for improving drug therapy in disabled elderly people. Isr Med Assoc J 2007;9:430-4.

14. Garfinkel D, Mangin D. Feasibility study of a systematic approach for discontinuation of multiple medications in older adults: addressing polypharmacy. Arch Intern Med 2010;170:1648-54.

15. Somers M, Rose E, Simmonds D, et al. Quality use of medicines in residential aged care. Aust Fam Physician 2010;39:413-16.

16. Rancourt C, Moisan J, Baillargeon L, et al. Potentially inappropriate prescriptions for older patients in long-term care. BMC Geriatr 2004;4:9.
17. Linsky A, Simon SR, Bokhour B. Patient perceptions of proactive medication discontinuation. Patient Educ Couns 2015;98: 220-5.

18. Flick U, Garms-Homolová V, Röhnsch G. "And mostly they have a need for sleeping pills": physicians' views on treatment of sleep disorders with drugs in nursing homes. J Aging Stud 2012;26: 484-94.

19. Harriman K, Howard L, McCracken R. Deprescribing medication for frail elderly patients in nursing homes: a survey of Vancouver family physicians. B C Med J 2014;56:436-41.

20. Anderson K, Stowasser D, Freeman C, et al. Prescriber barriers and enablers to minimising potentially inappropriate medications in adults: a systematic review and thematic synthesis. BMJ Open 2014;4:e006544.

21. Reeve E, To J, Hendrix I, et al. Patient barriers to and enablers of deprescribing: a systematic review. Drugs Aging 2013;30: 793-807.

22. Lim CJ, Kwong MW, Stuart RL, et al. Antibiotic prescribing practice in residential aged care facilities-health care providers' perspectives. Med J Aust 2014;201:98-102.

23. Hughes $\mathrm{CM}$, Lapane $\mathrm{K}$, Watson MC, et al. Does organisational culture influence prescribing in care homes for older people? A new direction for research. Drugs Aging 2007;24:81-93.

24. Halvorsen KH, Ruths S, Granas AG, et al. Multidisciplinary intervention to identify and resolve drug-related problems in Norwegian nursing homes. Scand J Prim Health Care 2010;28:82-8

25. Schuling J, Gebben H, Veehof LJ, et al. Deprescribing medication in very elderly patients with multimorbidity: the view of Dutch GPs. A qualitative study. BMC Fam Pract 2012;13:56

26. Reeve E, Wiese MD, Hendrix I, et al. People's attitudes, beliefs, and experiences regarding polypharmacy and willingness to deprescribe. J Am Geriatr Soc 2013;61:1508-14.

27. Scott IA, Denaro CP, Bennett CJ, et al. Towards more effective use of decision support in clinical practice: what the guidelines for guidelines Don't tell you. Intern Med J 2004;34:492-500.

28. Cantrill JA, Sibbald B, Buetow S. The Delphi and nominal group techniques in health services research. Int $J$ Pharm Pract 1996;4:67-74.

29. McMurray AR. Three decision-making aids: brainstorming, nominal group, and Delphi technique. J Nurs Staff Dev 1994;10:62-5.

30. Delbecq $A L$, Van de Ven $A H$. A group process model for problem identification and program planning. J Appl Behav Sci 1971;7:466-92.

31. Campbell SM, Cantrill JA. Consensus methods in prescribing research. J Clin Pharm Ther 2001;26:5-14.

32. Dening $\mathrm{KH}$, Jones $\mathrm{L}$, Sampson EL. Preferences for end-of-life care: a nominal group study of people with dementia and their family carers. Palliat Med 2013;27:409-17.

33. Van de $\mathrm{Ven} A \mathrm{H}$, Delbecq $\mathrm{AL}$. The nominal group as a research instrument for exploratory health studies. Am J Public Health 1972;62:337-42.

34. van Teijlingen E, Pitchforth E, Bishop C, et al. Delphi method and nominal group technique in family planning and reproductive health research. J Fam Plann Reprod Health Care 2006;32:249-52.

35. Gallagher M, Hares T, Spencer J, et al. The nominal group technique: a research tool for general practice? Fam Pract 1993;10:76-81.

36. Drennan V, Walters K, Lenihan $\mathrm{P}$, et al. Priorities in identifying unmet need in older people attending general practice: a nominal group technique study. Fam Pract 2007;24:454-60.

37. Romagnoli KM, Handler SM, Ligons FM, et al. Home-care nurses' perceptions of unmet information needs and communication difficulties of older patients in the immediate post-hospital discharge period. BMJ Qual Saf 2013;22:324-32.

38. Pitkälä $\mathrm{KH}$, Juola $\mathrm{AL}$, Kautiainen $\mathrm{H}$, et al. Education to reduce potentially harmful medication use among residents of assisted living facilities: a randomized controlled trial. J Am Med Dir Assoc 2014;15:892-8.

39. Farrell B, Tsang C, Raman-Wilms L, et al. What are priorities for deprescribing for elderly patients? Capturing the voice of practitioners: a modified delphi process. PLOS ONE 2015;10: e0122246.

40. Anthierens S, Tansens A, Petrovic M, et al. Qualitative insights into general practitioners views on polypharmacy. BMC Fam Pract 2010;11:65.

41. Gnjidic D, Le Couteur DG, Kouladjian L, et al. Deprescribing trials: methods to reduce polypharmacy and the impact on prescribing and clinical outcomes. Clin Geriatr Med 2012;28:237-53.

42. Castle NG. The influence of consistent assignment on nursing home deficiency citations. Gerontologist 2011;51:750-60. 
43. Koren MJ. Person-centered care for nursing home residents: the culture-change movement. Health Aff (Millwood) 2010;29:312-17.

44. Roberts T, Nolet K, Bowers B. Consistent assignment of nursing staff to residents in nursing homes: A critical review of conceptual and methodological issues. Gerontologist 2015;55:434-47.

45. Rahman A, Straker JK, Manning L. Staff assignment practices in nursing homes: review of the literature. J Am Med Dir Assoc 2009;10:4-10.

46. Iyer S, Naganathan V, McLachlan AJ, et al. Medication withdrawal trials in people aged 65 years and older: a systematic review. Drugs Aging 2008;25:1021-31.

47. Beer C, Loh PK, Peng YG, et al. A pilot randomized controlled tria of deprescribing. Ther Adv Drug Saf 2011;2:37-43.

48. Dalleur O, Boland B, Losseau C, et al. Reduction of potentially inappropriate medications using the STOPP criteria in frail older inpatients: a randomised controlled study. Drugs Aging 2014;31:291-8.

49. Nelson MR, Reid CM, Krum H, et al. Predictors of normotension on withdrawal of antihypertensive drugs in elderly patients: prospective study in second Australian national blood pressure study cohort. BMJ 2002;325:815.
50. Tannenbaum C, Martin P, Tamblyn R, et al. Reduction of inappropriate benzodiazepine prescriptions among older adults through direct patient education: the EMPOWER cluster randomized trial. JAMA Intern Med 2014;174:890-8.

51. Van Spall HG, Toren A, Kiss A, et al. Eligibility criteria of randomized controlled trials published in high-impact general medical journals: a systematic sampling review. JAMA 2007;297:1233-40.

52. Iden KR, Hjørleifsson S, Ruths S. Treatment decisions on antidepressants in nursing homes: a qualitative study. Scand J Prim Health Care 2011;29:252-6.

53. Schmidt I, Claesson CB, Westerholm B, et al. The impact of regular multidisciplinary team interventions on psychotropic prescribing in Swedish nursing homes. J Am Geriatr Soc 1998;46: 77-82.

54. Crotty M, Halbert J, Rowett $\mathrm{D}$, et al. An outreach geriatric medication advisory service in residential aged care: a randomised controlled trial of case conferencing. Age Ageing 2004;33:612-17.

55. Bain KT, Holmes HM, Beers MH, et al. Discontinuing medications: a novel approach for revising the prescribing stage of the medication-use process. J Am Geriatr Soc 2008;56: 1946-52. 